\title{
EFECTOS DE LAS CARACTERÍSTICAS FÍSICAS EN LOS INGRESOS LABORALES EN ECUADOR, 2012*
}

\author{
Recibido: 12 de agosto de 2016 • Aprobado: 27 de febrero de 2017 \\ DOI: $10.22395 /$ seec.v20n42a5 \\ Héctor Alberto Botello* \\ Isaac Guerrero Rincón ${ }^{* * *}$
}

\section{RESUMEN}

El objetivo del estudio es analizar el efecto que tienen las características físicas de los trabajadores sobre los salarios en el mercado laboral ecuatoriano. Empleando información de los microdatos de la Encuesta Nacional de Salud y Nutrición de Ecuador para el 2012, se estiman ecuaciones de Mincer en las que se relaciona el ingreso salarial por hora con las características socioeconómicas del trabajador. Como los datos provenientes de encuestas tienen un problema implícito nombrado sesgo de selección, que consiste a la ausencia de aleatoriedad en la escogencia de los individuos encuestados, se utiliza la ecuación anti-sesgo de Heckman para corregir este problema. Los resultados evidencian que a mayor estatura, peso o índice de masa corporal se obtienen mayores salarios. Estos resultados son consistentes incluso cuando se diferencian por género, área geográfica y nivel educativo.

\section{PALABRAS CLAVE}

Salarios; nivel salarial; diferencias salariales; atributos físicos; Ecuador.

\section{CLASIFICACIÓN JEL}

\author{
E24, J31
}

\section{CONTENIDO}

Introducción; 1. Metodología; 1.1 Modelo de Mincer; 1.2. Datos; 2. Resultados; 3. Conclusiones; 4. Bibliografía.

* El artículo de investigación es producto del proyecto "Capital social y Desarrollo", el cual se encuentra activo desde el año 2013. La investigación pertenece al grupo de Grupo de Investigación sobre Desarrollo Regional y Ordenamiento Territorial (GIDROT, reconocido por Colciencias en la categoría B en 2016), Facultad de Ciencias Humanas, Universidad Industrial de Santander, Bucaramanga, Colombia.

** Economista, Universidad Industrial de Santander, Bucaramanga, Colombia. Candidato a la maestría en ingeniería industrial, Universidad Industrial de Santander, Bucaramanga, Colombia. Estudiante de la Maestría en Ciencias Económicas, Universidad Nacional de Colombia, Bogotá, Colombia. Miembro del Grupo de Investigación sobre Desarrollo Regional y Ordenamiento Territorial, Universidad Industrial de Santander, Bucaramanga, Colombia. Dirección postal: Carrera 27 calle 9 ciudad universitaria, escuela de Economía, Universidad Industrial de Santander, Bucaramanga, Colombia. Teléfono: 3012829726. Correo electrónico: hbotellop@unal.edu.co.

*** Economista, Universidad Pedagógica y Tecnológica de Colombia, Tunja, Colombia. Magíster en Economía, Universidad Nacional de Colombia, Bogotá, Colombia. Profesor, Universidad Industrial de Santander, Bucaramanga, Colombia. Miembro del Grupo de Investigación sobre Desarrollo Regional y Ordenamiento Territorial, Universidad Industrial de Santander, Bucaramanga, Colombia. Dirección postal: Carrera 27 calle 9 ciudad universitaria, Universidad Industrial de Santander, Bucaramanga, Colombia. Teléfono: 3012829726. Correo electrónico: iguerrin@uis.edu.co. 


\section{EFFECTS OF PHYSICAL CHARACTERISTICS ON LABOR INCOME IN ECUADOR, 2012}

\section{ABSTRACT}

The goal of this paper is to analyze the effects that physical characteristics of workers have over wages in the Ecuadorian labor market. Using micro data information taken from the 2012 National Health and Nutrition Survey of Ecuador, Mincer equations are estimated relating the wage income per hour and socioeconomic characteristics of the worker variables. Given that survey data present an implicit problem called selection bias, which consists in lack of randomness within the survey respondents, a Heckman anti-bias equation is used in order to correct this issue. Results show that those taller and heavier individuals, as well as those that have higher body-mass, tend to have higher wages. These results are consistent even between genders, geographic areas and educational levels

\section{KEY WORDS}

Wages, wage level, wage diferences, physical attributes, Ecuador.

\section{JEL CLASSIFICATION}

E24, J31

\section{CONTENT}

Introduction; 1. Methodology; 1. 1Mincer Model; 1.2. Data; 2. Results; 3. Conclusions; 4. Bibliography.

\section{EFEITOS DAS CARACTERÍSTICAS FÍSICAS NOS INGRESSOS LABORAIS NO EQUADOR, 2012 \\ RESUMO}

O objetivo do estudo é analisar o efeito que tem as características físicas dos trabalhadores sobre os salários no mercado laboral equatoriano. Empregando informação dos micro-dados da Enquete Nacional de Saúde e Nutrição do Equador para 2012, se estimam equações de Mincerianas nas que se relaciona o ingresso salarial por hora com as características socioeconómicas do trabalhador. Como os dados provenientes de enquetes têm um problema implícito nomeado viés de seleção, que consiste na ausência de aleatoriedade na escolha dos indivíduos entrevistados, se utiliza a equação anti-viés de Heckman para corrigir este problema. Os resultados evidenciam que a maior estatura, peso ou índice de massa corporal se obtém maiores salários. Estes resultados são consistentes incluso quando se diferencia por gênero, área geográfica e nível educativo.

\section{PALAVRAS CHAVE}

Salários, nível salarial, diferenças salariais, atributos físicos, Equador.

\section{CLASSIFICAÇÃO JEL}

E24, J31

\section{CONTEÚDO}

Introdução; 1. Metodologia; 1.1 Modelo de Mincer; 1.2. Dados; 2. Resultados; 3. Conclusões; 4. Bibliografia. 


\section{INTRODUCCIÓN}

La discriminación en el mercado laboral ha sido tema frecuente en la literatura económica, y han sido identificadas algunas características socioeconómicas del individuo como fuertemente correlacionadas con el ingreso laboral, tales como el género, la edad, la ocupación, el sector económico, entre otras. Las investigaciones de Biddle y Hamermesh (1998); Hamermesh y Biddle (1994); Yamamura, Smyth y Zhang (2015) sugieren que las características físicas de los individuos también se encuentran relacionadas con las ganancias salariales

En este orden de ideas, el análisis de los determinantes de las brechas salariales asociadas a las características físicas resulta relevante en la formulación de políticas públicas, dado que la existencia de estos diferenciales desalienta el esfuerzo del trabajo y aumentan la probabilidad de que los individuos persistan en trabajos con baja remuneración. Asimismo, permitirá establecer mejores procesos de contratación en las empresas donde prime la capacidad productiva sobre factores subjetivos.

En este sentido, tres enfoques se han abordado para determinar cómo las características físicas influyen en la remuneración. El primero es cómo la apariencia influye en las ganancias laborales. Los estudios de Hamermesh y Parker (2003) sugieren que los profesores cuya belleza, medida por un índice que recoge las valoraciones subjetivas de sus alumnos, está por encima de la media ganan un 10 a 15 por ciento más en promedio. En segundo lugar, investigaciones como la de Heineck (2005) miden cómo el peso del individuo afecta el desempeño en el mercado laboral; esta aproximación ha surgido con interés a causa de la alta incidencia de obesidad dentro de los países.

En general, según Cawley (2000) la penalidad por obesidad existe, ya que estas personas tienen más posibilidades de ser tratadas como menos inteligentes al carecer de autodisciplina o motivación. En tercer lugar, se encuentran los autores como Komlos (1998) que incluyen la estatura como una variable de control adicional al peso. Estos consideran que la estatura refleja, en cierta medida, el grado de inversión en capital humano de los individuos en su salud, lo que mejora el rendimiento en el mercado de trabajo. No obstante, según la revisión literaria de Persico, Postlewaite y Silvema (2004) los efectos de la estatura en sí mismos han sido examinados por pocos estudios.

El alcance de estas investigaciones han sido los países desarrollados, dado que han contado con un número amplio de encuestas antropométricas de los individuos que se combinan con sus características en el mercado laboral. Mitra (2001) llega a la conclusión de que las mujeres más altas en ocupaciones gerenciales 
o profesionales reciben una prima salarial de alrededor del 2,5\% por cada pulgada adicional de estatura.

Schultz (2002) examina los efectos de las características físicas sobre los salarios para muestras de trabajadores de Ghana, Brasil y los EE. UU. Sus resultados sugieren primas salariales por cada centímetro adicional en la talla adulta de aproximadamente 1,5\% en Ghana y Brasil, y de alrededor de 0,4\% para los trabajadores estadounidenses. Sin embargo, en los países en desarrollo el avance de estas investigaciones se ha visto retrasado por la falta de información. Dentro de la literatura hallada para el caso latinoamericano, se encuentra el estudio de Thomas y Strauss (1997) quienes investigan en las áreas urbanas en Brasil; los resultados sugieren que la estatura es un determinante importante del salario tanto para hombres como para las mujeres. Esta relación también ha sido validada por otros trabajos, entre ellos Sohn (2015) para Indonesia, Gao y Smyth (2010) para China y Tao (2014) en Taiwán.

Con base en lo anterior, el presente trabajo desea contribuir a la literatura al analizar el efecto de las características físicas (estatura, peso e índice de masa corporal -IMC-) de los trabajadores sobre los salarios en Ecuador con base en la Encuesta Nacional de Salud y Nutrición (ENSANUT) del año 2012. Los resultados muestran que existe evidencia estadística que demuestra que cierta parte de las diferencias salariales puede ser explicada por las características físicas de los trabajadores. En términos empíricos existen primas a favor de los trabajadores con mayor peso, estatura o IMC.

El trabajo se divide en cuatro secciones incluyendo esta introducción; en la siguiente sección se detalla la metodología y datos a utilizar con la presentación de los resultados en la segunda sección; finalmente, en la última sección se exponen las conclusiones.

\section{METODOLOGÍA}

\subsection{Modelo de Mincer}

El primer modelo utilizado para estimar las brechas salariales entre grupos es el modelo de Mincer (1974), con este se estiman qué efectos posibles puede tener cada una de las características de los trabajadores sobre sus ingresos laborales.

Según Asplund y Pereira (1999) y Psacharopoulos y Patrinos (2004) en esta estimación se plantea como variable dependiente el logaritmo de la remuneración salarial por hora, y como variables independientes características socioeconómicas del trabajador (ecuación 1). En este sentido, el modelo se interpreta como semiloga- 
rítmico estimado por medio de mínimos cuadrados ordinarios (MCO); como insumo se utilizan datos transversales de las encuestas de empleo.

$$
\operatorname{Ln}(\mathrm{Y})=\beta_{0}+\beta_{1} \operatorname{Edu}+\beta_{2} \operatorname{Exp}+\beta_{3} \operatorname{Exp}^{2}+\beta_{4} \mathrm{~S}+\beta_{5} \mathrm{~L}+\varepsilon
$$

Donde:

- Y son el ingreso laboral por hora del individuo. Este incluye las prestaciones sociales derivadas del trabajo, así como las dotaciones en forma monetaria dadas en el empleo.

- Edu es el capital humano acumulado en forma de educación, medida por el nivel de escolaridad del individuo.

- $\quad$ Exp y Exp $p^{2}$ corresponde a la experiencia y su cuadrado; se aproxima por medio de la edad del trabajador.

- S hace referencia a las variables que miden las características físicas del trabajador, incluye la altura, el peso y el IMC.

- L corresponde a otras características del trabajador.

- $\varepsilon$ es el término de perturbación aleatoria que se distribuye según una normal.

Al modelo estimado se le deben realizar pruebas para la validación de los supuestos de normalidad de las variables, la homocedasticidad, la no colinealidad y la no omisión de variables del modelo; en caso de no cumplirse alguno de los supuestos anteriores se procederá a la re-estimación. Finalizado lo anterior, los los coeficientes ( $\beta$ ) estimados se interpretan como el cambio porcentual sobre el salario que ejercen cada una de las características de los trabajadores. El grado de validación del modelo se calcula mediante el porcentaje de la varianza de la variable dependiente que es captada por parte de las variables de control, indicador denominado $\mathrm{R}^{2}$; entre más alto, más efectivo es el modelo en determinar el comportamiento de la variable dependiente.

Para Esquivel (2007) y Rivera (2013), se encuentra que en el manejo de los datos mediante encuestas existe un problema implícito nombrado sesgo de selección. Este consiste en la ausencia de aleatoriedad en la escogencia de los individuos encuestados. El sesgo aparece dentro de la muestra, ya que la presencia aumentada de cierto grupo poblacional desvía las deducciones sobre las variables analizadas hacia las características de dicho grupo. La corrección de Heckman es la forma más implementada de arreglar este sesgo y consiste en utilizar variables instrumentales que permitan obtener estimadores consistentes en presencia de regresores en- 
dógenos. El método empieza con la estimación de una ecuación que determina la probabilidad de que el individuo participe en el mercado laboral utilizando como variables independientes los factores que pueden incidir en la elección:

$$
p_{i}=\beta 0+z_{i} \varphi+u_{i}
$$

Siendo $p_{i}$ la probabilidad de participar en el mercado laboral del individuo $i$, $z_{i}$ es un conjunto de variables que explican la decisión a participar en el mercado laboral del individuo $i, \phi$ es un vector de coeficientes, y u son los errores. Después, se incorpora la corrección del sesgo al modelo de Mincer anteriormente expuesto.

$$
\operatorname{Ln}(\mathrm{Y})=\beta_{0}+\beta_{1} \mathrm{Edu}+\beta_{2} \operatorname{Exp}+\beta_{3} \operatorname{Exp}^{2}+\beta_{4} \mathrm{~S}+\beta_{5} \mathrm{~L}+z_{i} \varphi+\varepsilon
$$

El resultado final es la estimación del cambio que sufre el ingreso laboral por hora frente a una variación en la estatura, el peso o el IMC del individuo. Estos indicadores han sido utilizados en varias ocasiones en la literatura para explicar las diferencias salariales gracias a su facilidad de cálculo y comparación, por ejemplo, en Biddle y Hamermesh (1998); y Yamamura, Smyth y Zhang (2015).

Sin embargo, Dinda et al. (2006) sugieren que existen diferentes formas de analizar estos efectos cambiando la naturaleza de las variables independientes que aproxima empíricamente tal fenómeno. En este sentido, el presente estudio explora cuatro tipos de efectos. Desde la primera hasta la tercera especificación se valora el efecto de la estatura, el peso y el IMC ${ }^{1}$ del individuo sobre el salario de los individuos como una variable continua. Después se configuran rangos de las variables en cuartiles para el peso y la estatura, además de los grupos indicados por la organización mundial de la salud para el IMC. Otras especificaciones no lineales con el peso, la estatura y sus interacciones fueron probadas, pero no fueron significativas.

Adicionalmente, en Mitra (2001) se recomienda realizar ejercicios de efectos marginales particulares entre grupos de interés, por ejemplo, el género, dado que entre hombres y mujeres existen diferencias biológicas que determinan sus características físicas e influyen posteriormente en su rendimiento en el mercado laboral. Adicionalmente, los individuos se discriminan por nivel educativo, dado que las exigencias en conocimientos de los empleos influyen en su remuneración.

1 Se calcula como el cociente entre el peso y el cuadrado de la estatura. 


\subsection{Los datos $^{2}$}

Los datos utilizados en esta investigación se tomaron de la Encuesta Nacional de Salud y Nutrición (ENSANUT) del Ecuador para el año 2012. El estudio original escogió una muestra de 19.949 viviendas en las 24 provincias del Ecuador, con una cobertura de las áreas rurales y urbanas con ayuda de 12 módulos. Si bien la encuesta presenta una preocupación por variables asociadas a la salud y la antropometría, también incluye módulos de calidad de vida donde se incluyen las actividades económicas de los individuos.

Por las condiciones de la investigación, se escogieron 19.159 ocupados entre los 16 y 59 años que habían completado los formularios antropométricos. Con los factores de expansión esta población es representativa de 3.409.951 personas. Dentro de las preguntas se utilizaron las cifras de peso y talla de los trabajadores medidos en centímetros para elaborar el índice de masa corporal como variable auxiliar en la aproximación de las características físicas del trabajador.

La tabla 1 muestra algunas estadísticas por grupos de interés dentro de la encuesta, tales como la media del ingreso laboral por hora en dólares corrientes, el peso, la talla y el IMC, promedio. En esta población, los hombres constituyen el 59 \% de los ocupados con un salario medio por hora de USD 1,89 mientras que para las mujeres es de USD 1,77; igualmente, los hombres son levemente más altos que las mujeres y, en consecuencia, más pesados.

Por su parte, los trabajadores urbanos (73\%) tienen salarios significativamente más altos frente a los rurales (USD 2,01 vs USD 1,44) junto con medidas de peso y talla más elevadas. En términos de etnias (indígenas, afrodescendientes, montubios), estas ocupan porcentajes de alrededor el $16 \%$ de la población y presentan menores salarios. Es interesante resaltar los menores niveles en términos de peso y talla que presenta la población indígena ecuatoriana. Finalmente, la mayor parte de la población ocupada (80 \%) tiene un nivel de educación inferior a Secundaria, a la vez que se observa que a mayor capital humano (grados de escolaridad) aumenta el salario.

2 Los autores suministrarán la base de datos construida y utilizada en el estudio a cualquier investigador interesado, mediante solicitud al correo electrónico: hbotellop@unal.edu.co. 
Héctor Alberto Botello - Isaac Guerrero Rincón

Tabla 1. Estadísticas descriptivas de los datos

\begin{tabular}{|c|c|c|c|c|c|c|}
\hline Variable & Característica & N..$^{\circ}$ Personas & $\begin{array}{l}\text { Talla } \\
(m)\end{array}$ & Peso $(k g)$ & IMC & $\begin{array}{c}\text { Media } \\
\text { Ingreso por } \\
\text { hora (USD) }\end{array}$ \\
\hline \multirow{2}{*}{ Sexo } & Hombre & 2.022 .408 & 1,59 & 67,22 & 26,70 & 1,89 \\
\hline & Mujer & 1.387 .377 & 1,57 & 63,81 & 25,94 & 1,77 \\
\hline \multirow{2}{*}{ Área } & Urbano & 2.500 .931 & 1,65 & 70,75 & 25,84 & 2,01 \\
\hline & Rural & 908.854 & 1,53 & 62,93 & 26,96 & 1,44 \\
\hline \multirow{4}{*}{ Etnia } & Indígena & 189.293 & 1,53 & 59,92 & 25,47 & 1,34 \\
\hline & Afro & 148.396 & 1,61 & 70,69 & 27,15 & 1,68 \\
\hline & Montubio & 196.056 & 1,59 & 67,22 & 26,46 & 1,64 \\
\hline & Resto & 2.876 .040 & 1,58 & 66,38 & 26,52 & 1,9 \\
\hline \multirow{4}{*}{$\begin{array}{l}\text { Nivel de } \\
\text { Educación }\end{array}$} & Primario & 1.171 .342 & 1,58 & 67,52 & 26,90 & 1,47 \\
\hline & Secundario & 1.536 .397 & 1,58 & 67,94 & 26,27 & 1,84 \\
\hline & Superior & 666.683 & 1,61 & 69,89 & 26,86 & 2,87 \\
\hline & Postgrado & 35.363 & 1,61 & 71,10 & 27,27 & 6,17 \\
\hline \multirow{4}{*}{ Nivel IMC } & Bajo peso & 42.152 & 1,62 & 46,37 & 17,60 & 1,49 \\
\hline & Normal & 1.221 .585 & 1,61 & 58,50 & 22,60 & 1,67 \\
\hline & Obesidad & 1.423 .902 & 1,60 & 70,00 & 27,28 & 1,93 \\
\hline & Sobrepeso & 703.854 & 1,58 & 84,36 & 33,51 & 1,92 \\
\hline
\end{tabular}

Fuente: Encuesta Nacional de Salud y Nutrición (ENSANUT), INEC, 2012

Dentro de los ocupados ecuatorianos se observa la prevalencia de la obesidad según el aumento de la edad (gráfico 1). En las edades de 16 a 20 años, las personas obesas por IMC alcanzaron el $5 \%$, mientras que en los mayores de $53 \%$ el porcentaje alcanza el 29 \%. Por nivel educativo, la distribución de los grupos por IMC se mantiene.

Con la base de datos se procedió a aplicar los métodos econométricos por mínimos cuadrados ordinarios (MCO) para conocer la influencia de las características físicas en el salario de los trabajadores. 
Gráfico 1. Porcentaje de personas por grupos del índice de masa corporal (IMC)

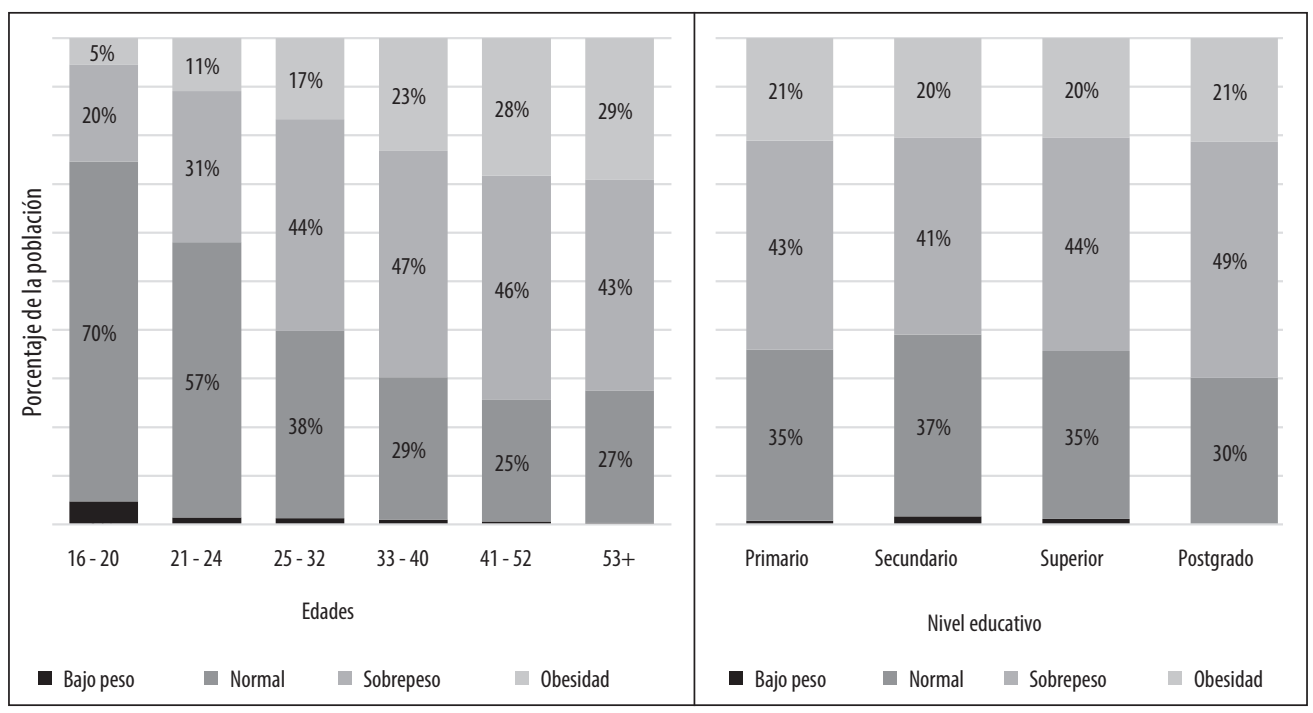

Fuente: Encuesta Nacional de Salud y Nutrición (ENSANUT), INEC, 2012

\section{RESULTADOS}

La Tabla 2 presenta los principales resultados de las estimaciones del modelo de Mincer especificado en la sección anterior en la ecuación $1^{3}$. Se incluyen en esta tabla las observaciones totales, el $\mathrm{R}^{2}$ y las variables tanto continuas como categóricas y el efecto porcentual que tienen sobre el ingreso laboral promedio cada variable incluida en el modelo. Después de refinar la base de datos y extraer las observaciones perdidas, se estudiaron los ingresos laborales de cerca de 3,4 millones de trabajadores. El porcentaje de varianza estimado por el modelo de Mincer es cercano al $31 \%$, siendo todas las especificaciones en promedio igualmente eficientes.

Por su parte, el efecto de las variables continuas se lee como el cambio porcentual que tendrá la independiente en la variable dependiente; por ejemplo, un aumento del $1 \%$ en la edad de la trabajadora incrementa en un 2,7 \% el ingreso laboral promedio. Por otro lado, en las características categóricas, el cambio porcentual sobre el puntaje se da con relación a una característica base, por ejemplo, las mujeres ganan en promedio un $7,2 \%$ menos que los hombres.

Sobre la tesis central de este trabajo, las especificaciones donde las variables de características físicas son continuas señalan una ganancia positiva del 0,4\%, 0,3 \% y $0,5 \%$ por cada unidad adicional de IMC, peso y talla que posean los individuos, respectivamente.

3 El total de las estimaciones se encuentra en el anexo. 
Por cuartiles de rango y peso, estas relaciones mono-tónicamente crecientes se mantienen, a excepción de los coeficientes por rangos de IMC, que muestran que las ganancias en salarios no son uniformes, ya que los salarios de las personas con peso normal, según IMC, son un 8,4 \% más altos que las personas con bajo peso, un $12,4 \%$ para las personas con sobrepeso y $11,5 \%$ adicional para personas con obesidad. Es decir, que hasta cierto punto los empleadores premian las condiciones de salud de sus trabajadores al observar mejores grados de peso proporcionalmente a la estatura.

Tabla 2. Resultado de las estimaciones por tipo de especificación. Mínimos cuadrados ordinarios con corrección de Heckman y errores robustos

\begin{tabular}{|c|c|c|c|c|c|}
\hline Variable & Característica evaluada & Coef. & Error & $\mathrm{P}>|z|$ & Característica base \\
\hline IMC & Continua & $0,4 \%$ & 0,00 & 0,00 & Continua \\
\hline Peso & Continua & $0,3 \%$ & 0,00 & 0,00 & Continua \\
\hline Talla & Continua & $0,5 \%$ & 0,00 & 0,00 & Continua \\
\hline \multirow{3}{*}{ Rangos IMC } & Normal & $8,4 \%$ & 0,00 & 0,00 & \multirow{3}{*}{ Bajo peso } \\
\hline & Sobrepeso & $12,9 \%$ & 0,00 & 0,00 & \\
\hline & Obesidad & $11,5 \%$ & 0,00 & 0,00 & \\
\hline \multirow{3}{*}{ Rangos de talla } & 2 Cuartil & $5,2 \%$ & 0,00 & 0,00 & \multirow{3}{*}{1 Cuartil } \\
\hline & 3 Cuartil & $8,0 \%$ & 0,00 & 0,00 & \\
\hline & 4 Cuartil & $12,1 \%$ & 0,00 & 0,00 & \\
\hline \multirow{3}{*}{ Rangos de peso } & 2 Cuartil & $3,6 \%$ & 0,00 & 0,00 & \multirow{3}{*}{1 Cuartil } \\
\hline & 3 Cuartil & $6,8 \%$ & 0,00 & 0,00 & \\
\hline & 4 Cuartil & $9,4 \%$ & 0,00 & 0,00 & \\
\hline
\end{tabular}

\begin{tabular}{|l|c|}
\hline R2 & 0,3085 \\
\hline Observaciones totales & 9.449 .398 \\
\hline Censuradas & 6.040 .085 \\
\hline No censuradas & 3.409 .313 \\
\hline Wald chi2(81) & 1.370 .000 \\
\hline Prob $>$ chi2 & 0,00 \\
\hline $\begin{array}{l}\text { Test LR test para la independencia de las ecuaciones de corrección del sesgo y estimación final } \\
\text { (rho }=0 \text { ): chi2(1) }=1883,05 . \text { Prob }>\text { chi2 }=0,0000\end{array}$ \\
\hline
\end{tabular}

Fuente: Cálculos de los autores con base en la Encuesta Nacional de Salud y Nutrición (ENSANUT), INEC, 2012 
Esta situación se repite cuando se evalúan discrecionalmente los salarios estimados por género y área de la ciudad (gráfica 2). Se aprecia que los hombres ganan más que las mujeres en todos los ámbitos, pero también muestran la misma curva ascendente en relación al IMC.

Gráfica 2. Ingreso laboral por hora estimado por sexo y rango del IMC. Áreas urbana y rural

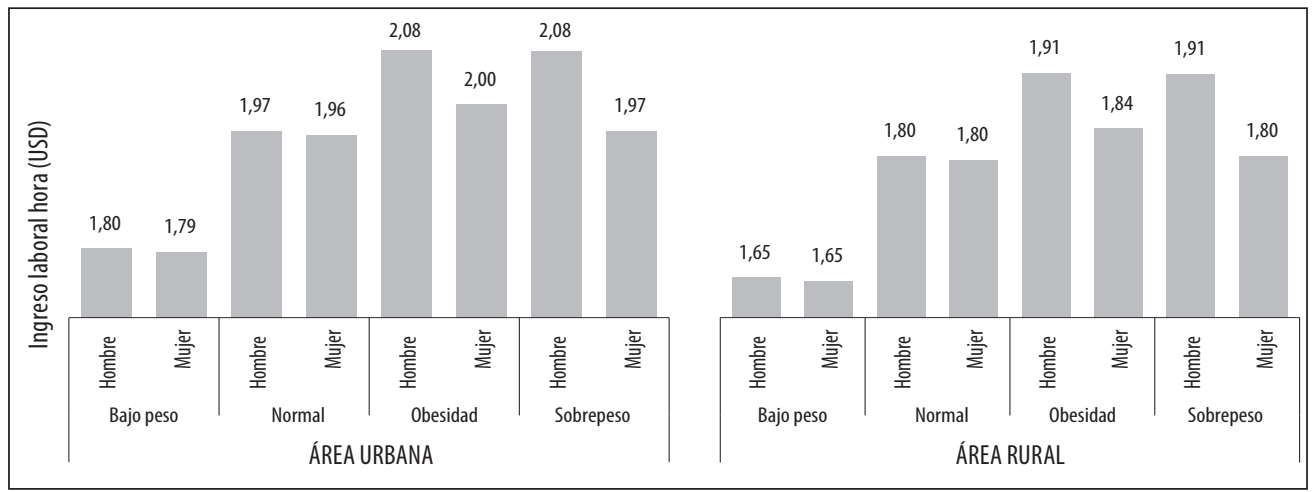

Fuente: cálculos de los autores con base en la Encuesta Nacional de Salud y Nutrición (ENSANUT), INEC, 2012

La discriminación por nivel educativo (gráfica 3) evidencia que mayor capital humano trae mejores rendimientos en el mercado laboral. Cuando se interactúan los rangos el IMC con cada nivel educativo, no existen pérdidas o ganancias dentro de cada nivel, pero se mantienen las encontradas en forma agregada entre grado educativo. Sin embargo, en los niveles de posgrado, la presencia de sobrepeso en los trabajadores sí se castiga con una penalidad salarial del $4.5 \%$ frente a los de bajo peso.

Gráfica 3. Ingreso laboral por hora estimado por nivel educativo y rango del IMC

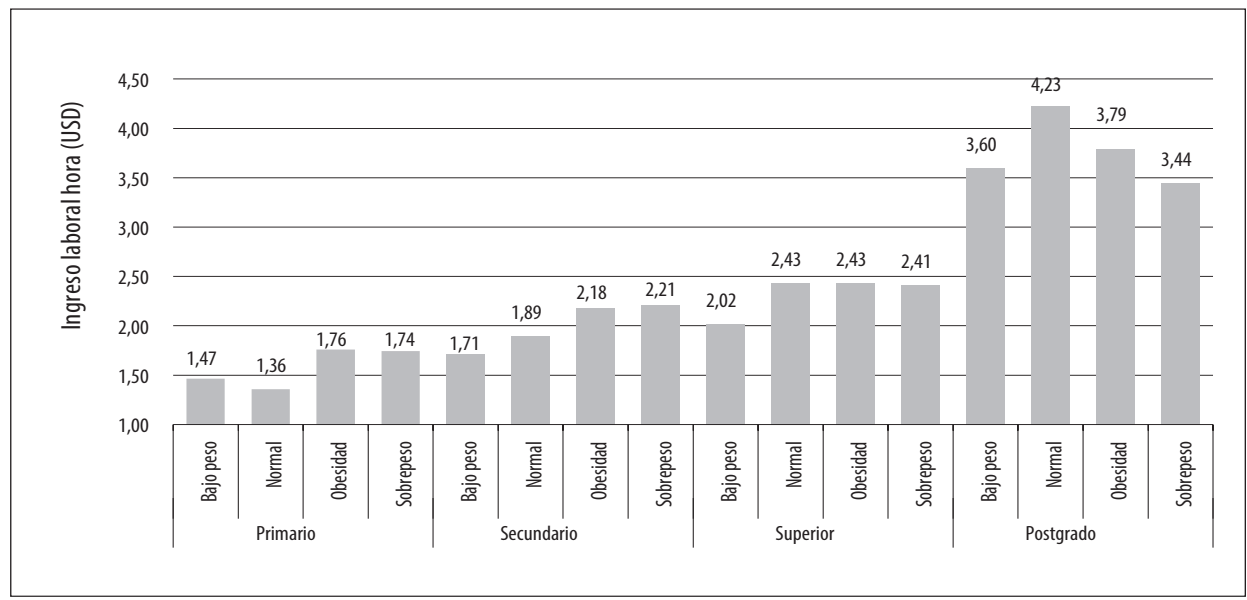

Fuente: Cálculos del autor con base en la Encuesta Nacional de Salud y Nutrición (ENSANUT), INEC, 2012 
Sobre las estimaciones se obtuvieron otros resultados de interés (anexo):

- La educación es la mejor inversión que pueden hacer los individuos para incrementar su ingreso laboral por hora. En este orden de ideas, por cada grado adicional de educación, el salario de la persona se incrementa en 3,4 \%. Estos resultados van en la línea de lo obtenido para Ecuador por Rivera (2013), Espinoza y Sánchez (2009) donde la ganancia educativa se encuentra entre el 4 \% y el 5 \%.

- Las mujeres ganan en promedio menos que los hombres (-7,2\%), según explican Espinoza y Sánchez (2009); la causa de la discriminación es dada por menos horas de trabajo a las que se ven expuestas por su trabajo doméstico. Rivera (2013), por su parte, afirma que en 2012 la brecha de género en Ecuador alcanzaba el $10 \%$ y remarca la tendencia decreciente en esta diferencia desde 2007.

- Los resultados también sugieren que el salario se incrementa con la edad (2,7 $\%)$ pero con una tasa decreciente $(0,01 \%)$.

- Se observa que las personas que se definen como indígenas reciben menores salarios que el resto de la población $(-7,5 \%)$

- Los sectores financieros e industriales ofrecen los mejores ingresos laborales, con salarios un $14 \%$ y 6,2 \% por encima de la agricultura.

\section{CONCLUSIONES}

En esta investigación se analizaron los efectos de la estatura, el peso y el IMC en los ingresos laborales de los hombres y las mujeres en Ecuador, utilizando algunas variables como control para corregir el sesgo de selección. Los resultados muestran que existe evidencia suficiente para afirmar que existen diferencias salariales explicadas por las características físicas de los trabajadores. En términos empíricos reciben salarios más altos los trabajadores con mayor peso o estatura. Asimismo, las personas con más IMC también reciben mejores remuneraciones, que son estas más altas cuando se logra un grado de sobrepeso. Estas diferencias se mantienen incluso controlando por género, región y nivel educativo ${ }^{4}$.

Los resultados anteriores sugieren que la inversión (pública o privada) para la mejora de la atención de la salud del niño es importante para la creación de capital humano de la salud, que es esencial para garantizar rendimientos futuros. En términos de políticas públicas en relación con el mercado laboral, los resultados de este trabajo apuntan a que muchas veces los empleadores no usan criterios

4 Como apunte, el estudio de Chen (2012) sugiere que el peso a una edad en particular establecerá el perfil de ganancias de por vida de un individuo específico. 
objetivos basados en la productividad de los trabajadores, sino subjetivos acerca del estado físico y la salud.

Este estudio tiene limitaciones, por ejemplo, la restringida capacidad del modelo para predecir la totalidad de la varianza de los ingresos laborales que están en función de variables no observables. Estas incluyen habilidades interpersonales, adaptabilidad social, características de la empresa, la motivación etc. Más datos detallados sobre los factores del mercado laboral que generalmente permanecen sin ser observados pueden facilitar una mejor comprensión de cómo se desarrolla la penalización salarial por las características físicas.

\section{BIBLIOGRAFÍA}

Asplund, R. y Pereira P. (1999). Returns to Human Capital in Europe. A Literature Review. The Research Institute of the Finnish Economy, Helsinki, 368p.

Biddle, J. y Hamermesh, D., (1998). Beauty, productivity and discrimination: lawyers' look and lucre. En: Journal of Labor Economics, Vol. 16, No. 1, p. 172-201.

Cawley, J. (2000). An instrumental variables approach to measuring the effect of body weight on employment disability. En: Health Services Research, Vol. 35, No. 5 (Pt 2), p. 1159-1179.

Chen, A. (2012). Cover image When does weight matter most? En: Journal of Health Economics Vol. 31, No. 1, p. 285-295.

Dinda, S.; Gangopadhyay, P. K.; Chattopadhyay, B. P.; Saiyed, H. N.; Pal, M. y Bharati, P. (2006). Height, weight and earnings among coalminers in India. En: Economics y Human Biology, Vol. 4, No. 3, p. 342-350.

Espinoza, N., y Sánchez, L. (2009). Estimación de la brecha salarial entre hombres y mujeres: un análisis por cuantiles para el Ecuador. En Económicas Escuela Superior Politécnica del Litoral (ESPOL). Vol. 4. p. 2-20.

Esquivel, V. (2007). Género y Diferenciales de Salarios en la Argentina, p. 363-392. En: M. Novick y H. Palomino (edits.) Estructura productiva y empleo: un enfoque transversal. Buenos Aires, Argentina: Ministerio de Trabajo, Empleo y Seguridad Social. ISBN 978-84-96571-68-6, 397p.

Gao W., Smyth R. (2010). Health human capital, height and wages in China. En: The Journal of Development Studies, Vol. 46, No. 3, p. 466-484.

Hamermesh, D.S. y Biddle, J., (1994). Beauty and the labor market. En: The American Economic Review, Vol. 84, No. 5, p. 1174-1194.

Hamermesh, D.S. y Parker, A. (2003). Beauty in the classroom: professors' pulchritude and putative pedagogical productivity. En: NBER Working Paper, No. 9853, 21p.

Heckman, J. (1979). Sample selection bias as a specification error. En: Econometrica, Vol. 47 No 1, p.153-161.

Heineck, G. (2005). Up in the skies? The relationship between body height and earnings in Germany. En: Labour, Vol. 19, No. 3, p. 469-489. 
Komlos, J., (1998). Shrinking in a growing economy? The mystery of physical height during the industrial revolution. En: The Journal of Economic History, Vol. 58, No. 3, p. 779-803.

Mincer, J. (1974). Schooling, Experience and Earnings. En National Bureau of Economic Research, ISBN: 0-870-14265-8. 187p.

Mitra, A. (2001). Effects of physical attributes on the wages of males and females. En: Applied Economics Letters, Vol. 8, No. 11, p. 731-735.

Persico, N.G.; Postlewaite, A. y Silveman, D. (2004). The effect of adolescent experience on labour market outcomes: the case of height. En: Journal Political Economy, Vol. 112, No. 5, p. 1019-1053.

Psacharopoulos, G. y Patrinos H. (2004). Returns to investment in education: a further update. En: Education economics, Vol 12, No 2, p. 111-134.

Rivera, J. (2013). Teoría y práctica de la discriminación en el mercado laboral ecuatoriano (20072012). En: Analítika. Vol. 5, No 1, p. 3-18.

Schultz, P.T. (2002). Wage gains associated with height as a form of health human capital En: Economic Growth Center Discussion Paper. No. 841, 26p.

Sohn K. (2015). The height premium in Indonesia. En: Economics \& Human Biology, Vol. 16, enero, p. 1-15.

Tao H.L. (2014). Height, weight, and entry earnings of female graduates in Taiwan. En: Economics in Human Biology, Vol. 13, marzo, p. 85-98.

Thomas, D. y Strauss, J. (1997). Health and wages: Evidence on men and women in urban Brazil. En: Journal of Econometrics, Vol. 77, No. 1, p. 159-185.

Yamamura, E.; Smyth, R. y Zhang, Y. (2015). Decomposing the effect of height on income in China: The role of market and political channels. En: Economics in Human Biology, Vol.19, diciembre, p. 62-74. 
Efectos de las características físicas en los ingresos laborales en Ecuador, 2012

\section{ANEXO. RESULTADO COMPLETO DE LAS ESTIMACIONES}

\begin{tabular}{|c|c|c|c|c|c|}
\hline Variable & $\begin{array}{c}\text { Característica } \\
\text { evaluada }\end{array}$ & Coef. & Error & $\mathrm{P}>|z|$ & Característica base \\
\hline $\begin{array}{l}\text { Años de educa- } \\
\text { ción }\end{array}$ & Continua & $3,4 \%$ & 0,00 & 0,00 & Continua \\
\hline Sexo & Mujer & $-7,2 \%$ & 0,00 & 0,00 & Hombre \\
\hline Edad & Continua & $2,7 \%$ & 0,00 & 0,00 & Continua \\
\hline $\mathrm{Edad}^{\wedge} 2$ & Continua & $-0,01 \%$ & 0,00 & 0,00 & Continua \\
\hline Sector ciudad & Rural & $-12,1 \%$ & 0,00 & 0,00 & Urbano \\
\hline \multirow{23}{*}{ Provincia } & Bolivar & 211 o & O 01 & קח ת ח & A \\
\hline & $\begin{array}{l}\text { Bollvar } \\
\text { Cañar }\end{array}$ & $-11,1 \%$ & 0,00 & 0,00 & Azuay \\
\hline & Carchi & $-13,7 \%$ & 0,00 & 0,00 & \\
\hline & Cotopaxi & $-8,0 \%$ & 0,00 & 0,00 & \\
\hline & Chimborazo & $-14,0 \%$ & 0,00 & 0,00 & \\
\hline & El Oro & $-3,1 \%$ & 0,00 & 0,00 & \\
\hline & Esmeraldas & $-16,3 \%$ & 0,00 & 0,00 & \\
\hline & Guayas & $-5,3 \%$ & 0,00 & 0,00 & \\
\hline & Imbabura & $-8,3 \%$ & 0,00 & 0,00 & \\
\hline & Loja & $-4,1 \%$ & 0,00 & 0,00 & \\
\hline & Los Ríos & $-8,4 \%$ & 0,00 & 0,00 & \\
\hline & Manabí & $-7,4 \%$ & 0,00 & 0,00 & \\
\hline & Morona & $1,1 \%$ & 0,00 & 0,03 & \\
\hline & Napo & $-0,4 \%$ & 0,01 & 0,58 & \\
\hline & Pastaza & $-2,6 \%$ & 0,01 & 0,00 & \\
\hline & Pichincha & $4,1 \%$ & 0,00 & 0,00 & \\
\hline & Tungurahua & $-6,7 \%$ & 0,00 & 0,00 & \\
\hline & $\begin{array}{l}\text { Zamora Chin- } \\
\text { chipe }\end{array}$ & $0,4 \%$ & 0,01 & 0,54 & \\
\hline & Galápagos & $5,8 \%$ & 0,01 & 0,00 & \\
\hline & Sucumbíos & $-3,9 \%$ & 0,00 & 0,00 & \\
\hline & Orellana & $7,3 \%$ & 0,01 & 0,00 & \\
\hline & Santo domingo & $-5,9 \%$ & 0,00 & 0,00 & \\
\hline & Santa Elena & $-17,1 \%$ & 0,00 & 0,00 & \\
\hline & & & & & \\
\hline \multirow{7}{*}{$\begin{array}{l}\text { Tipo de ocupa- } \\
\text { ción }\end{array}$} & $\begin{array}{l}\text { Empleado } \\
\text { privado }\end{array}$ & $-39,9 \%$ & 0,00 & 0,00 & Empleado público \\
\hline & Jornalero o peón & $-43,8 \%$ & 0,00 & 0,00 & \\
\hline & Patrono(a) & $6,0 \%$ & 0,00 & 0,00 & \\
\hline & Socio(a) & $-33,3 \%$ & 0,00 & 0,00 & \\
\hline & Cuenta propia & $-32,9 \%$ & 0,00 & 0,00 & \\
\hline & No remunerado & $-76,1 \%$ & 0,01 & 0,00 & \\
\hline & Doméstico & $-43,2 \%$ & 0,00 & 0,00 & \\
\hline
\end{tabular}


Héctor Alberto Botello - Isaac Guerrero Rincón

\begin{tabular}{|c|c|c|c|c|c|}
\hline Variable & $\begin{array}{c}\text { Característica } \\
\text { evaluada }\end{array}$ & Coef. & Error & $\mathrm{P}>|z|$ & Característica base \\
\hline \multirow{9}{*}{$\begin{array}{l}\text { Rama de activi- } \\
\text { dad }\end{array}$} & Minería & $-5,2 \%$ & 0,00 & 0,00 & Agricultura \\
\hline & Industria & $6,2 \%$ & 0,00 & 0,00 & \\
\hline & Electricidad & $0,8 \%$ & 0,00 & 0,00 & \\
\hline & Construcción & $3,1 \%$ & 0,00 & 0,00 & \\
\hline & Comercio & $3,8 \%$ & 0,00 & 0,00 & \\
\hline & Financieros & $14,3 \%$ & 0,00 & 0,00 & \\
\hline & Administrativos & $-6,1 \%$ & 0,00 & 0,00 & \\
\hline & Público & $-4,9 \%$ & 0,00 & 0,00 & \\
\hline & Sociales & $-2,9 \%$ & 0,00 & 0,00 & \\
\hline & & & & & \\
\hline \multirow{3}{*}{ Grupo étnico } & Afroecuatoriano & $4,3 \%$ & 0,00 & 0,00 & Indígena \\
\hline & Montubio & $11,1 \%$ & 0,00 & 0,00 & \\
\hline & $\begin{array}{l}\text { Resto de la Po- } \\
\text { blación }\end{array}$ & $7,9 \%$ & 0,00 & 0,00 & \\
\hline \multirow[t]{3}{*}{ Lugar de trabajo } & Fuera del hogar & $12,4 \%$ & 0,00 & 0,00 & Dentro del hogar \\
\hline & & & & & \\
\hline & Constante & $33,5 \%$ & 0,01 & 0,00 & \\
\hline
\end{tabular}

\section{R2 por especificación}

\begin{tabular}{|l|r|}
\hline 1 & 0,3085 \\
\hline 2 & 0,3082 \\
\hline 3 & 0,3092 \\
\hline 4 & 0,3095 \\
\hline
\end{tabular}

\begin{tabular}{|l|r|}
\hline Observaciones totales & 9.449 .398 \\
\hline Censuradas & 6.040 .085 \\
\hline No censuradas & 3.409 .313 \\
\hline Wald chi2(81) & 1.370 .000 \\
\hline Prob $>$ chi2 & 0,00 \\
\hline \multicolumn{2}{|l|}{$\begin{array}{l}\text { Test LR test para la independencia de las ecuaciones de corrección del sesgo y estimación final } \\
\text { (rho }=0): \text { chi2(1) }=1883,05 . \text { Prob }>\text { chi2 }=0,0000\end{array}$} \\
\hline
\end{tabular}

